\title{
Phenotypic cofirmatory disc diffusion test (PCDDT), double disc synergy test (DDST), E-test OS diagnostic tool for detection of extended spectrum beta lactamase (ESBL) producing Uropathogens
}

\begin{abstract}
Background: The aim of this study was to probe the best diagnostic tool for the detection of Extended Spectrum $\beta$-lactamase $(E S \beta L)$ producing uropathogen and their antimicrobial susceptibility profile to treat the infections properly.

Methods: Clinical samples of urine were cultured on Cysteine Lactose Electrolyte Deficient (CLED) Agar medium. Antimicrobial sensitivity tests were carried out by Kirby-Bauer disc diffusion method. Phenotypic methods were used for further confirmation of $\beta$ - lactamase production by phenotypic confirmatory disc diffusion test (PCDDT), double disc synergy test (DDST) and by E-test for ESBL production. Aswab on Mueller-Hinton (MH) agar plates was used for further studies and the Optical Density (O.D.) of the cultures was set to 0.1 (at $530 \mathrm{~nm}$ ).
\end{abstract}

Results: Sample size N= 200 was selected from patients suffering from UTI. Out of the 200 samples, $\mathrm{n}=141$ samples yielded Aerobic Gram Negative Bacteria (AGNB). The commonest organism isolated was E.coli $\mathrm{n}=108$, best antimicrobial result of $95 \%$ was shown by imipenem. Among the AGNB isolates, 20 organisms (12.98\%) were ESBL producers. E.coli showed highest ESBL production of $85 \%$. The most effective antimicrobial in ESBL producers was Imipenem (84\%) Augmentin was least sensitive $(05 \%)$.

Conclusion: ESBL production is a common phenomenon in UTI patients and screening by DDST for these enzymes is a good epidemiological tool to assess the overall situation in a certain setup.It was also seen that Imipenem (Carbapenem) is the drug of choice.

Keywords: ESßL, CLED, DDST, AGNB isolates, imipenem
Volume 3 Issue 3 - 2017

\author{
Rashid Iqbal,' Nadeem Ikram, ${ }^{2}$ Muhammad \\ Shoaib, ',2 Muhammad Javaid asad,' Raja Tahir \\ Mehmood,' Abida Niazi, ${ }^{3}$ Anam Asghar, ${ }^{4}$ \\ Bushra Ishfaq, ${ }^{4}$ Faiza Naseer ${ }^{4}$ \\ 'Department of Biochemistry, Arid Agriculture University, \\ Pakistan \\ ${ }^{2}$ Department of Medical Technology, Shifa Tameer e Millat \\ University Islamabad, Pakistan \\ 3University of Veterinary and Animal Sciences Lahore, Pakistan \\ ${ }^{4}$ Government College University Faisalabad, Pakistan
}

\section{Correspondence: Faiza Naseer, Government college university Faisalabad, Islamabad, Pakistan, Tel 92-33336665I5, Email faiza.naseer@ymail.com}

Received: February 22, 2017| Published: June 16, 2017
Abbreviations: ES $\beta$ L, extended spectrum $\beta$-lactamase; CLED, cysteine lactose electrolyte deficient; PCDDT, phenotypic confirmatory disc diffusion test; $\mathrm{MH}$, mueller-hinton; OD, optical density; AGNB, aerobic gram negative bacteria; UTI, urinary tract infection; OPD, outpatient door; COD, casualty outdoor; DDST, double disc synergy test

\section{Introduction}

Urinary tract infection (UTI) is one of the most common problems for people to get hospitalized or get medical advice and is one of the most common nosocomial infections. Examination of bacterial spectrum and antibiotics sensitivity is necessary for the empirical treatment of nosocomial urinary tract infections. ${ }^{1}$ Different aerobic Gram-negative bacteria (AGNB) produce beta lactamases which cause resistance to beta-lactam agents. ${ }^{2}$ ESBLs were discovered in 1980 , nursing homes and hospitals are found as main reservoirs of extended spectrum beta- lactamase (ESBL) producing bacteria. Hospital patients serve as a reservoir for these resistant organisms. ${ }^{3-5}$ Enterobacteriacae produce ESBL sand are globally serious nosocomial pathogens. ${ }^{67}$ ESBLs producing organisms are clinically important and remain a major cause of treatment failure with Cephalosporin's and other classes of antibiotics in the world. ${ }^{8}$
Third generation of Cephalosporin's was introduced in 1980, which brought a relief in the fight against beta-lactamase resistance to antibiotics. Cephalosporin's were introduced due to increased resistance by beta-lactamases produced by different Gram-negative bacteria such Klebsiella pneumoniae, E.coli, Pseudomonas aeruginosa, Proteus spp. and may disseminate new host, for example, produced by Neisseria gonorrhea and Hemophillus influenza. ${ }^{9}$ ESBLs are plasmid or chromosomal mediated $\beta$-lactamases ( $\beta$-lactamases are enzymes that cleave the $\beta$-lactam ring) of already existing mutant $\beta$-lactamases (SHV-1, TEM-1 and TEM-2) as a result of frequent use of third generation cephalosporin's and aztreonam. ${ }^{10,11}$ Clavulanic acid or sulbactum are used as inhibitors of ESBLs. ${ }^{5}$ However Amp-c lactamase overproduction and porin loss result in the production of inhibitor resistant ESBLs. ${ }^{12}$ The severity and location of infection, liver function, kidney function, geographical resistance and the presence of implants will determine the efficacy of antibiotics. It is also observed that the effectiveness of antibiotics also depend upon the lactation and age of pregnancy. ${ }^{2}$

\section{Materials and methods}

\section{Collection of Samples}

Urine samples of symptomatic UTI were identified by conventional 
techniques. Samples of urine were collected from midstream clean catch, transurethral catheterization, plastic bag, or supra-pubic aspiration according to clinical status and age of patient. $\mathrm{N}=200$ was selected for this study.

i. Inclusion Criteria: All indoor and Outpatient Door (OPD) samples from UTI patients were included irrespective of age and sex.

ii. Exclusion Criteria: All clinical urine samples for routine examination and other than UTI patients were excluded.

\section{Sample processing}

Clinical samples of urine were collected from indoor, Casualty outdoor (COD) and OPD patients at Microbiology Laboratory Department and were cultured on CLED Agar and incubated for 24 hours at $37^{\circ} \mathrm{C}$. Cultures of bacteria were processed for subculturing, Gram staining and Biochemical tests for identification. Antimicrobial sensitivity testing was carried out by Kirby-Bauer disc diffusion method and Phenotypic Confirmatory Disc Diffusion Test (PCDDT), Double Disc Synergy Test (DDST) and E-Test was For ESBL detection.

\section{Identification of isolates}

Identification of all aerobic gram negative bacteria (E.coli, Klebsiellaspp, Pseudomonas aeruginosa, Proteusspp.) was performed by morphological characteristics, Gram staining, biochemical tests and specific disc pattern for ESBL production. Different biochemical tests were performed on the isolates for confirmation of all aerobic gram negative bacterial colonies. Known controls were also put up with each biochemical test. ${ }^{13}$

\section{Indole production test}

Pseudomonas was indole negative and E.coli was indole positive. ${ }^{13}$

i. MR-VP Test: E.coli was MR positive and VP negative while Klebsiella spp was MR negative and VP positive. ${ }^{13}$

ii. Citrate Utilization Test: Positive control was Klebsiella pneumoniae and negative control was E.coli ATCC 25922. ${ }^{13}$

iii. Oxidase Test: Positive control was P. aeruginosa ATCC 27853 and negative control E. coli ATCC 25922. ${ }^{13}$

\section{Analytical profile index (API) 20E}

Non-conclusive samples were run on API and were interpreted according to API chart. API $20 \mathrm{E}$ is known as a standardized identification system for gram negative rodsand Entero bacteriacae. It has 20 biochemical parameters. Bacterial suspension was prepared equal to $0.5 \mathrm{McF}$ arland index .A homogeneous bacterial suspension was achieved by careful emulsification. Strips wells were charged with bacterial suspension. Tubes were filled according to the test requirement. $\mathrm{H}_{2} \mathrm{~S}, \mathrm{ADH}, \mathrm{LDC}$, Urea and $\mathrm{ODC}$ tests were incubated with overlaying mineral oil. To check the quality of suspension, a purity plate was prepared by inoculation from the suspension. After 18 to 24 hours at $35 \pm 2^{\circ} \mathrm{C}$ incubation, add reagents in respective wells. Seven digits numerical profile was determined after positive results and which was looked up in API code comparable to organism identification ${ }^{13}$ (Table 1).

Table I Interpretation of biochemical tests on API 20E strip

\begin{tabular}{|c|c|c|c|c|}
\hline \multirow{2}{*}{ Test } & \multirow{2}{*}{ Active ingredients } & \multirow{2}{*}{ Reactions/enzymes } & \multicolumn{2}{|l|}{ Result } \\
\hline & & & Negative & Positive \\
\hline ONPG & 2-nitrophenyl- $\beta D$-galactopyranoside & beta-galactosidase & Colorless & Yellow \\
\hline $\mathrm{ADH}$ & L-arginine & Arginine dihydrolase & Yellow & Red/orange \\
\hline LDC & L-lysine & Lysine decarboxylase & Yellow & Red/orange \\
\hline ODC & L-ornithine & Ornithine decarboxylase & Yellow & Red/orange \\
\hline CIT & Trisodium citrate & Citrate utilization & Pale green/yellow & Blue-green/blue \\
\hline $\mathrm{H} 2 \mathrm{~S}$ & Sodium thiosulfate & $\mathrm{H} 2 \mathrm{~S}$ production & Colorless & Blackdeposit/thin line \\
\hline URE & Urea & Urease & Yellow & Red/orange \\
\hline TDA & L-tryptophane & Tryptophane deaminase & Yellow & TDA/immediate. I Reddishbrown \\
\hline IND & L-tryptophane & Indole production & Colorless & Jammes/immediate.2Pink \\
\hline VP & Sodium pyruvate & Acetoin production & Colorless & $V_{p} I+V_{p} / I 0$ min.3Pink/red \\
\hline GEL & Gelatin & Gelatinase & No diffusion & Diffusion of black pigment \\
\hline GLU & D-glucose & F/O4, glucose & Blue-green/blue & Yellow \\
\hline MAN & D-mannitol & $\mathrm{F} / \mathrm{O}$,mannitol & Blue-green/blue & Yellow \\
\hline INO & Inositol & F/O,inositol & Blue-green/blue & Yellow \\
\hline SOR & D-sorbitol & F/O,sorbitol & Blue-green/blue & Yellow \\
\hline RHA & L-rhamnose & F/O,rhamnose & Blue-green/blue & Yellow \\
\hline SAC & D-sucrose & $\mathrm{F} / \mathrm{O}$,saccharose & Blue-green/blue & Yellow \\
\hline MEL & D-melibiose & F/O,melibiose & Blue-green/blue & Yellow \\
\hline AMY & Amygdalin & F/O,amygdalin & Blue-green/blue & Yellow \\
\hline ARA & L-arabinose & $\mathrm{F} / \mathrm{O}$,arabinose & Blue-green/blue & Yellow \\
\hline
\end{tabular}

Citation: Iqbal R, Ikram N, Shoaib M, et al. Phenotypic cofirmatory disc diffusion test (PCDDT), double disc synergy test (DDST), E-test OS diagnostic tool for detection of extended spectrum beta lactamase (ESBL) producing Uropathogens.J Appl Biotechnol Bioeng. 2017;3(3):344-349. 


\section{ESBL screening using PCDDT, DDST and e test as diagnostic tool}

Phenotypic methods were used for further confirmation of $\beta$ lactamase production for all the isolates showing resistance to $3^{\text {rd }}$ generation cephalosporin, namely Ceftazidime, Cefotaxime and Ceftriaxone. Swab on Mueller-Hinton (MH) agar plates was used for further studies and the Optical Density (O.D.) of the cultures was set to 0.1 (at $530 \mathrm{~nm}$ ). The screening was done according to CLSI instructions. ${ }^{14}$

\section{Phenotypic Confirmatory Disc Diffusion Test (PCD- DT) For ESBL Detection}

A combination of clavulanic acid $(10 \mathrm{mcg})$ and ceftazidime (30 $\mathrm{mcg}$ ) was used. Both discs were placed on Muller Hinton agar plates which were earlier swabbed by respective culture and incubated for $24 \mathrm{hrs}$. At $37^{\circ} \mathrm{C}$. More than $5 \mathrm{~mm}$ increase in the zone diameter for Ceftazidime- Clavulanic acid was considered positive ESBL production $^{14}$ (Figure 1).

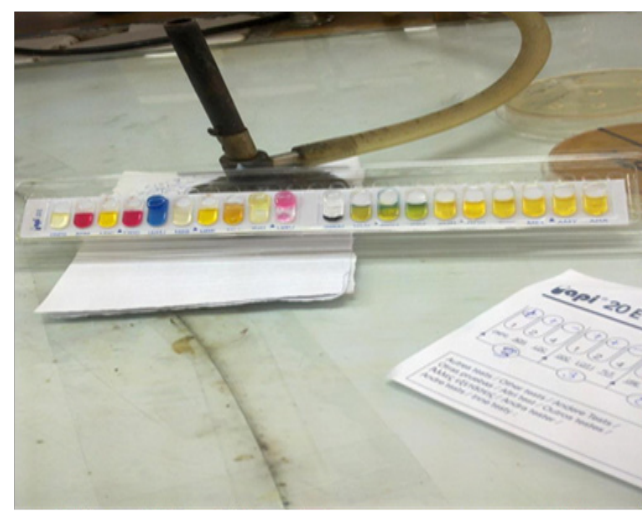

Figure I Extended spectrum $\beta$-lactamase.

\section{Double Disc Synergy Test (DDST)}

Five antibiotics were used for DDST namely Aztreonam (30 mcg), Amoxicillin-Clavulanic acid (20/10 mcg), Ceftriaxone (30 mcg), Ceftazidime (30 mcg) and Cefotaxime ( $30 \mathrm{mcg})$. At center AmoxicillinClavulanic acid disc was placed and these discs were placed at a distance of $1.5 \mathrm{~cm}$. Development of the zone of inhibition towards the Clavulanate disc at $37^{\circ} \mathrm{C}$ after $24 \mathrm{hrs}$ incubation was indicative of a potential ESBL positive organism ${ }^{15}$ (Figure 2).

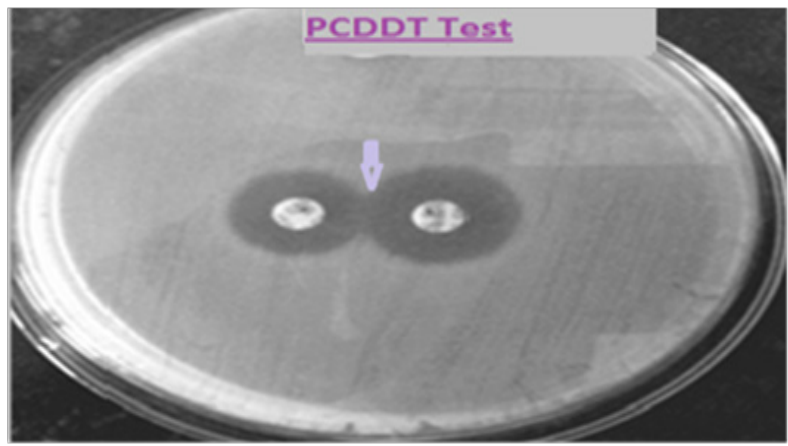

Figure 2 Phenotypic confirmatory disc diffusion test.

\section{Confirmation of ESBL Production by E-Test}

Multi-Enzyme (Ezy) Strips were used for confirmation of ESBL production. These strips differ from the conventional E-strips in that they contain a gradient of 3 antibiotics with and without Clavulanic acid on either sides of the strip respectively instead of one antibiotic and so these strips are different from conventional strips. The MultiEzy Strips have Cefotaxime, Cefipime and Ceftazidime (Noted as MIX side) on one side in a two-fold gradient and the same antibiotics with Clavulanic acid (Noted as MIX+side) on the other side. A ratio of inhibition zones of MIX and MIX + for $\geq 8 \mathrm{~mm}$ was found as a positive E-test $^{14}$ (Figure 3).

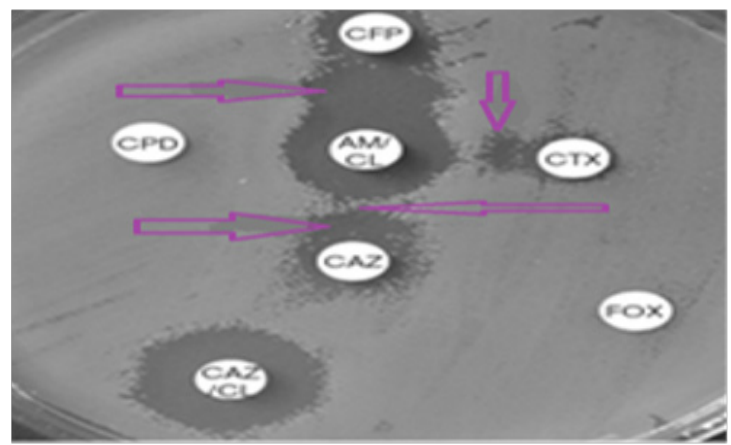

Figure 3 Double disc synergy test.

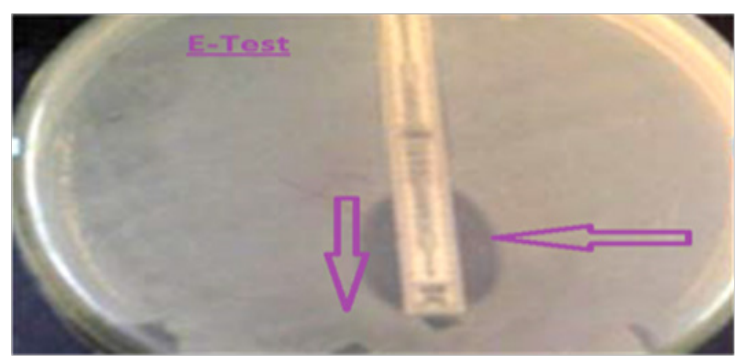

Figure 4 Confirmation of ESBL by E-test.

\section{Results}

Two hundred samples $\mathrm{N}=200$ were selected for current study, samples were collected from patients suffering from urinary tract infection (UTI). N=141 were AGNB. We got 154 different pathogenic strains (some samples yielded double and triple growths). The most common organism isolated was E.coli i.e. n $=108$, followed by Coliform spp, Klebsiellaspp, Pseudomonas aeruginosa and Proteus sppin an order $\mathrm{n}=22, \mathrm{n}=12 \mathrm{n}=04$ and $\mathrm{n}=03$ out of 154 pathogenic strains yielded from 141 positive samples respectively. Organism's breakup and percentages are given in Table 2 . Of the $\mathrm{N}=154$, best results of $95 \%$ shown by imipenem, $84 \%$ and $80 \%$ sensitivity were seen in case of Tazocin and Amikacin respectively. Augmentin showed the poorest sensitivity of $6 \%$. Individual details of the susceptibility pattern of the applied panel of antibiotics are shown in Table 3 .

Table 2 Overall organisms identified

\begin{tabular}{ll}
\hline Organisms & No of Isolates N=I 54 \\
\hline Enterococcus & $05(3.5 \%)$ \\
E.coli & $108(70 \%)$ \\
Klebsiellaepneumoniae & $12(08 \%)$ \\
Pseudomonas aeruginosa & $04(2.5 \%)$ \\
Coliform & $22(14.28 \%)$ \\
Proteus spp & $03(02 \%)$ \\
Total & $154(100 \%)$ \\
\hline
\end{tabular}


Table 3 Overall sensitivity pattern

\begin{tabular}{|c|c|c|c|}
\hline Antibiotics & Sensitive & Intermediate & Resistant \\
\hline Amikacin(AK)(applied in 93cultures) & (74) $80 \%$ & (05) $05 \%$ & (I4) $15 \%$ \\
\hline Augmentin(AUG)(154) & (09) $06 \%$ & (0I) $0.6 \%$ & (I44) $93.4 \%$ \\
\hline Tazocin(TZP)(86) & (72) $84 \%$ & (08) $09 \%$ & (06) $07 \%$ \\
\hline Cefepime (FEP)(07) & (02) $28 \%$ & (0I) $14 \%$ & (04) $58 \%$ \\
\hline Ceftazidime(CAZ)(96) & (23) $24 \%$ & (02) $02 \%$ & (7I) $74 \%$ \\
\hline Ceftriaxone(CRO)(98) & (20) $20 \%$ & (02) $02 \%$ & (78) $78 \%$ \\
\hline Ciprofloxacin(CIP)(76) & (I5) $20 \%$ & (06) $08 \%$ & (55) $72 \%$ \\
\hline Imepenem(IMI) II5 & (109) 95\% & (03) $2.5 \%$ & (03) $2.5 \%$ \\
\hline Gentamycin $(\mathrm{CN})(30)$ & (I3) $43 \%$ & (0I) $03 \%$ & (16) $54 \%$ \\
\hline Nitrofurantoin $(F)(110)$ & $(82) 75 \%$ & (05) $04 \%$ & (23) $21 \%$ \\
\hline Tobramycin(TOB)(05) & (0I) $20 \%$ & (0I) $20 \%$ & (03) $60 \%$ \\
\hline Suxzone(CES)(29) & (23) $79 \%$ & (0I) $3.5 \%$ & (05) $15 \%$ \\
\hline Chloramphenicol(C)(5) & (02) $40 \%$ & (0I) $20 \%$ & (02) $40 \%$ \\
\hline Fosfomycin(FOS)(I8) & (04) $22 \%$ & (0I) $06 \%$ & (13) $72 \%$ \\
\hline Cefotaxime(CTX)(37) & (13) $35 \%$ & (0I) $2.7 \%$ & (23) $62.3 \%$ \\
\hline Moxyfloxacin(MXF)(5I) & (19) $37 \%$ & (0I) $02 \%$ & (3I) $61 \%$ \\
\hline Nalidixic Acid(NA)(18) & (0I) $5.5 \%$ & (0I) $5.5 \%$ & (16) $89 \%$ \\
\hline Ampicillin(AMP)(06) & (0I ) $17 \%$ & (0I) $17 \%$ & (04) $66 \%$ \\
\hline Sulphmethazole((SXT)(29) & (03) $10 \%$ & (0I) $4 \%$ & (25) $86 \%$ \\
\hline Teicoplanin(TEC)(07) & (05) $72 \%$ & (0I) $14 \%$ & (0I) $14 \%$ \\
\hline Vancomycin(VAN)(05) & (03) $60 \%$ & (0I ) $20 \%$ & (0I) $20 \%$ \\
\hline Pipedinic Acid(PIP)(37) & (07) $19 \%$ & (0I) $03 \%$ & (29) $78 \%$ \\
\hline Meropenem(MRP)(09) & (07) $78 \%$ & (0I) $11 \%$ & (0I) $20 \%$ \\
\hline Polymixin $\mathrm{B}(\mathrm{PB})(05)$ & (03) $60 \%$ & (0I) $20 \%$ & (0I) $20 \%$ \\
\hline
\end{tabular}

Among the AGNBs isolates, $\mathrm{n}=20$ (12.98\%) were ESBL producers. These organisms included E.coli, Enterococcus, Coliform and Proteus spp. E.coli showed highest ESBL production of 17(85\%). Lesser degree of ESBL production was also seen in case of Proteus spp (05\%), Coliform spp (05\%) and Enterococcus (05\%) (Table 4). The most effective antimicrobial in ESBL producers was Imipenem (84\%) followed by Amikacin and Tazocin respectively. Augmentin was least sensitive $(05 \%)$ as shown in Table 5. Non conclusive isolates, which were not identified by cultural characteristics and Gram staining, were run on API20E Figure 1. PCDDT was used as one of the confirmatory tests for ESBL production as recommended by CLSI. The Ceftazidime discs alone or in combination with clavulanic acid were applied on Muller Hinton (MH) agar plates having the inoculation of test strain. A $5 \mathrm{~mm}$ increase in the zone of inhibition of the combination discs or ceftazidime alone were considered as positive ESBL production test. The test stain is inoculated on Muller Hinton agar plates. Amoxiclave/ Clavulanic acid disc was applied in the center of agar plate while Ceftazidmie, Ceftriaxone, Cefotaxime and Cefixime were applied at a distance of $20 \mathrm{~mm}$ from center to center from each other. The plates were incubated at. An enhancement of the zone of inhibition greater than $5 \mathrm{~mm}$ on the amoxiclave side of the disc were considered as ESBL producers. The test organism was inoculated on $\mathrm{MH}$ agar on which
E-test strips were applied. The plates were incubated at $37^{\circ} \mathrm{C}$ for 16-18hours. A ratio of inhibition zones of TZ and TZL for $\geq 8 \mathrm{~mm}$ was found as a positive E test Escherichia coli ATCC 25922 was used as the negative control and ESBL producing Klebsiella pneumoniae ATCC 700603 was used as the positive control throughout the study.

Table 4 Overall distribution of ESBL positive isolates $(\mathrm{N}=20)$.

\begin{tabular}{ll}
\hline Isolates & ESBL positive \\
\hline Enterococcus & $01(05 \%)$ \\
E.coli & $17(85 \%)$ \\
Coliform & $01(05 \%)$ \\
Proteus spp & $01(05 \%)$ \\
Total & $20(100 \%)$ \\
\hline
\end{tabular}


Table 5 Susceptibility pattern of ESBL producers: $(\mathrm{N}=20)$

\begin{tabular}{llll}
\hline Antibiotics & Sensitive & Intermediate & Resistant \\
\hline Amikacin(AK)(used in 20cultures) & $(16) 80 \%$ & $(02) 10 \%$ & $(02) 10 \%$ \\
Augmentin(AUG)(20) & $(0 I) 5 \%$ & $(01) 05 \%$ & $(18) 90 \%$ \\
Tazocin(TZP)(20) & $(15) 75 \%$ & $(03) 15 \%$ & $(02) 10 \%$ \\
Cefepime(FEP)(06) & $(02) 33.34 \%$ & $(01) 16.66 \%$ & $(03) 50 \%$ \\
Ceftazidime(CAZ)(20) & $(0) 0 \%$ & $(02) 10 \%$ & $(18) 90 \%$ \\
Ceftriaxone(CRO)(20) & $(0) 0 \%$ & $(03) 15 \%$ & $(17) 85 \%$ \\
Ciprofloxacin(CIP)(20) & $(06) 30 \%$ & $(02) 10 \%$ & $(14) 70 \%$ \\
Imepenem(IMI)(19) & $(16) 84.21 \%$ & $(02) 10.52 \%$ & $(01) 5.26 \%$ \\
Polymixin B(PB)(20) & $(11) 55 \%$ & $(04) 20 \%$ & $(05) 25 \%$ \\
\hline
\end{tabular}

\section{Discussion}

expensive. Whereas, PCDDT method is useful in busy diagnostic

The development and discovery of antibiotics was no doubt one of the greatest achievements of modern medicine. Unfortunately the appearance of bacterial resistance to antibiotics is frightening for the efficacy of many antimicrobial agents due to which hospital stay of the patients has increased and as a result great increase in the economic burden found. ${ }^{16}$ In this study, maximal numbers of ESBL producing organisms were derived from indoor patients. This could be due to the fact that infections caused by nosocomial organisms in admitted patients are more common than in OPD \& COD. The continuous exposure to the hospital environment plus the fact that the patients are on antibiotics or have prosthetic devices makes them more susceptible to infection. ${ }^{17} \mathrm{~A}$ similar finding was demonstrated by Supriya et al. ${ }^{17}$

In this study the commonest organism isolate was E.coli i.e. $70 \%$, followed by coliform 14\%, Klebsiella spp (8\%), Enterococcus 3.5\%, Pseudomonas aeruginosa $2.5 \%$, and Proteus spp (2\%). Although predominant organisms vary from one institution to another. This difference could be attributed by differences in geographical locations and standards of hygiene. ${ }^{2}$ In this study the high level of resistance to Augmentin may be as a result of inadvisable use of these drugs in the study population which lead to high selection pressure of resistant bacteria. Prescription of these drugs is common; therefore the use of these prescribed drugs in our settings is questionable. ${ }^{18}$ These are widely used because they are more affordable than other antibiotics. Fluor quinolones are effective agents for treatment of gram negative bacterial infection and resistance to ciprofloxacin is an early warning sign. ${ }^{8}$ In our country irrational and indiscriminate antibiotic usages as well as lack of effective antibiotic policies at all levels of treatment are the main contributory factors towards growing antimicrobial resistance. Mortality, morbidity and cost of treatment have considerably risen because of these resistant bacteria. ${ }^{19}$

Some Microbiology Laboratories may fail to detect ESBL positive organisms and can sometimes erroneously detect isolates to be sensitive to any of the third generation cephalosporin's (3GC) leading to therapeutic failures. Therefore, two more drugs are added to the routine panel of antimicrobial drugs for the regular detection of ESBL by PCDDT method. Every laboratory where molecular methods cannot be performed, should perform this technically simple PCDDT method, as this method is highly sensitive and specific compared to genotypic methods. ${ }^{20}$ To detect ESBL isolates many other methods like E Tests and Double Disc Synergy Test (DDST) commercially available, besides PCDDT. But these cannot be used routinely are

Microbiology laboratories, technically easy, reproducible, sensitive and cost effective. ${ }^{20}$

In order to quantify the synergy between extended-spectrum cephalosporin's and clavulanate, E-tests have been developed. The E-tests called CT/CTL, TZ/TZL and PM/PML are two-sided strips containing gradients of cefotaxime (CT), or ceftazidime (TZ) or cefepime (PM), either alone (at one end of the strip), or combined with clavulanate $4 \mathrm{mg} / \mathrm{L}$ (on the other end). The When the MIC value of the tested drug is reduced by more than three doubling dilution steps (MIC ratio $\geq 8$ ) in the presence of clavulanate, ESBL test is considered as positive. ${ }^{21}$ This test is also considered as positive when (a) a deformation of the CT, TZ or PM inhibition ellipse at the tapering end (b) or when there is either a rounded zone (phantom zone) just below the lowest concentration of CTL, TZL or PML gradients,. ESBL production is indicated by the presence of a phantom zone or an ellipse deformation. Interpreting results of the ESBL E-test strips requires training and is delicate. In a recent study, it has been reported that laboratories may fail to interpret correctly the inhibition ellipse in $30 \%$ of cases. ${ }^{22}$ In addition; ESBL detection by E-test may fail when the MIC values for cephalosporins fall outside the range of MICs available on the test strip. ${ }^{23}$ Double-disk synergy test (DDST) was the first test specifically designed to detect ESBL production in Enterobacteriaceae. ${ }^{24}$ It was initially designed to differentiate between cefotaxime-resistant strains, i.e., those overproducing cephalosporinase, and those producing ESBLs. The test is performed on agar with a $30-\mu \mathrm{g}$ disk of cefotaxime (and/or ceftriaxone and/or ceftazidime and/or aztreonam) and a disk of amoxicillin-clavulanate (containing $10 \mu \mathrm{g}$ of clavulanate) positioned at a distance of $30 \mathrm{~mm}$ (center to center), i.e., at the distance provided by several types of disk-dispenser. The test is considered as positive when a decreased susceptibility to cefotaxime is combined with a clear-cut enhancement of the inhibition zone of cefotaxime in front of the clavulanatecontaining disk, often resulting in a characteristic shape zone referred to as 'champagne-cork' or 'keyhole'. The DDST was first used in epidemiological studies to assess the spread of ESBL-producing Enterobacteriaceae in French hospitals..$^{25,26}$ It has been shown to work well with a wide range of Enterobacteriaceae species and ESBL types, and it is generally regarded as a reliable method for the detection of ESBLs, although it is sometimes necessary to adjust the disk spacing. It is important to note that reducing the distance between the clavulanatecontaining disk and the third-generation cephalosporin disk (e.g., to $20 \mathrm{~mm}$ ) significantly improves the test sensitivity. ${ }^{27,28}$ Since the antibiotic disks are routinely spaced $30 \mathrm{~mm}$ apart by several types of 
marketed disk dispenser, it is necessary, when the result of the test is equivocal (i.e., clear decrease in susceptibility to third-generation cephalosporins without clear synergy), to perform an additional test by arranging the disks by hand with narrower distances. ${ }^{28}$

\section{Conclusion}

In our set up, $12.9 \%$ isolates were ESBL producers. ESBL production is a common phenomenon in UTI patients and screening by DDST for these enzymes is a good epidemiological tool to assess the overall situation in a certain setup. It was also seen that Imipenem (Carbapenem) is the drug of choice. Hand washing still can reduce the spread of ESBL. Avoid prolong hospitalization wherever possible. Remove catheter/needles and other prosthetics as early as possible. Need for formulation of appropriate hospital antibiotics policy to avoid misuse and overuse of antibiotics. Formulation of effective infection control policy. Educate hospital staff against danger of cross infection. The knowledge of the resistance pattern of bacterial strains in a geographical area will help to guide the appropriate antibiotic use and such institutional studies will help to formulate an empirical antibiotic policy to treat Gram negative infections.

\section{Acknowledgements}

None.

\section{Conflict of interest}

The author declares no conflict of interest.

\section{References}

1. Wagenlehner FME, Niemetz A, Dalhoff A, et al. Spectrum and antibiotic resistance of uropathogens from hospitalized patients with urinary tract infections. Int J Antimicrob Agents. 2002;19(6):557-564.

2. Aggarwal R, Chaudhary U, Sikka R. Detection of extended spectrum beta-lactamase production among uropathogens. J Lab Physicians. 2004;1(1):7-10.

3. Wiener J, Quinn JP, Bradford PA, et al. Multiple antibiotic-resistant Klebsiella and Escherichia coli in nursing homes. JAMA. 1999;281(6):517523.

4. Arpin C, Dubois V, Coulange L, et al. Extendedspectrum B-lactamase producing Enterobacteriaceae in community and private health care centres. Antimicrob Agents Chemother. 2003;47(11):3506-3514.

5. Bradford PA. Extended Spectrum of $\beta$-Lactamases in the $21^{\text {st }}$-century: characterization, epidemiology and detection of this important resistant threat. Clin Microbiol Rev. 2001;14(4):933-951.

6. Sanders C, Sander WJ. Beta-lactam resistance in Gram-negative bacteria. Clin Infect Dis. 1992;15(5):824-883.

7. Moland ES, Black JA, Ourada J, et al. Occurrence of newer beta-lactamases in KlebsiellaPneumoniae isolates from 24 U.S. Hospitals. Antimicrob Agents Chemother. 2002;46(12):3837-3842.

8. Kliebe C, Nies BA, Meyer JF, et al. Evolution of plasmidcoded resistance to broad spectrum cephalosporins. Antimicrob Agents Chemother. 1995;28(2):302-307.

9. Paterson D. The epidemiological profile of infections with multidrug resistant Pseudomonas aeruginosa and Acinetobacter species. Clin Infect Dis. 2006;43(Suppl 2):43-48.

10. Shukla I, Tiwari R, Agrawal M. Prevalence of extended spectrum $\beta$-lactamase producing Klebsiella pneumoniae in a tertiary care hospital. Indian J Med Microbiol. 2004;22(2):87-91.
11. Giriyapur RS, Nandihal NW, Krishna BV, et al. Comparison of discdiffusion methods for the detection of extended spectrum beta lactamase-producing Enterobacteriaceae. J Lab Physicians. 2011;3(1):33-36.

12. Ananthan S, Subha A. Cefoxitinresistancemediated by loss of a porin in clinical strains of Klebsiella pneumoniae and Escherichia coli. Indian J Med Microbiol. 2005;23(1):20-23.

13. Harley JP, Prescott LM. Biochemical activities of bacteria. Laboratory exercise in microbiology. 2002;5:125-141.

14. CLSI. Performance standards for antimicrobial disc susceptibility tests. CLSI. 2011;31(1):100-121.

15. Jacoby GA, Medeiros AA. More Extended Spectrum beta lactamases. Antimicrob Agents Chemother. 1991;35(9):1697-1704.

16. Azap OK, Arslan H, Serefhanoğlu K, et al. Risk factors for extended-spectrum b-lactamase positivity in uropathogenic Escherichia coli isolated from community-acquired urinary tract infections. Clin Microbiol Infect. 2009;16(2):147-151.

17. Tankhiwale SS, Jalgaonkar SV, Ahamad S, et al. Evaluation of extended spectrum beta lactamase in urinary isolates. Indian $J$ Med Res. 2004;120(6):553-556.

18. Falagas ME, Karageorgopoulos DE. Extended-spectrum b-lactamase-producing Organisms. J of Hospital Infection. 2009;73:345-354.

19. Jabeen K, A Zafar, R Hasan. Frequency and sensitivity pattern of Extended Spectrum Beta Lactamase producing isolates in a tertiary care hospital laboratory of Pakistan. J Pak Med Assoc. 2002;55(10):215-220.

20. Kurokawa H, Yagi $\mathrm{T}$, Shibata $\mathrm{N}$, et al. worldwide proliferation of carbapenem-resistant gram negative bacteria. The Lancet. 1999;354(9182):955-960.

21. Cormican MG, Marshall SA, Jones RN. Detection of extended-spectrum beta-lactamase (ESBL)-producing strains by the E-test ESBL screen. J Clin Microbiol. 1996;34(8):1880-1884.

22. Leverstein-van Hall MA, Fluit AC, Paauw A, et al. Evaluation of the E-test ESBL and the BD Phoenix, VITEK 1, and VITEK 2 automated instruments for detection of extended-spectrum beta-lactamases in multiresistant Escherichia coli and Klebsiella spp. J Clin Microbiol. 2002;40(10):3703-3711

23. Linscott AJ, Brown WJ. Evaluation of four commercially available extended-spectrum beta-lactamase phenotypic confirmation tests. J Clin Microbiol. 2005;43(3):1081-1085.

24. Jarlier V, Nicolas MH, Fournier G, et al. Extended broad-spectrum beta-lactamases conferring transferable resistance to newer beta-lactam agents in Enterobacteriaceae: hospital prevalence and susceptibility patterns. Rev Infect Dis. 1988;10(4):867-878.

25. Legrand P, Fournier G, Bure A, et al. Detection of extended broad-spectrum beta-lactamases in Enterobacteriaceae in four French hospitals. Eur J Clin Microbiol Infect Dis. 1989;8(6):527-529.

26. Buré A, Legrand P, Arlet G, et al. Dissemination in five French hospitals of Klebsiella pneumoniae serotype K25 harbouring a new transferable enzymatic resistance to third generation cephalosporins and aztreonam. Eur J Clin Microbiol Infect Dis. 1988;7(6):780-782.

27. Thomson K, Sanders C. Detection of extended-spectrum beta-lactamases in members of the family Enterobacteriaceae: comparison of the double-disk and three-dimensional tests. Antimicrob Agents Chemother. 1992;36(9):1877-1882

28. Tzelepi E, Giakkoupi P, Sofianou D, et al. Detection of extended-spectrum beta-lactamases in clinical isolates of Enterobacter cloacae and Enterobacter aerogenes. J Clin Microbiol. 2000;38(2):542-546. 